\section{The important role of interdisciplinary collaboration in the management of a melanocytic skin lesion}

\author{
Anna Balato, ${ }^{1}$ Annunziata Raimondo, ${ }^{1}$ \\ Mariateresa Cantelli, ${ }^{1}$ Maria Siano, \\ Serena Lembo, ${ }^{1}$ Massimiliano Scalvenzi, ${ }^{1}$ \\ Nicola Balato' \\ 'Department of Dermatology, University \\ of Naples Federico II, Naples, Italy; \\ 2Department of Biomorphological and \\ Functional Sciences, Pathology Section, \\ School of Medicine, University Federico II \\ of Naples, Naples, Italy
}

\section{Abstract}

One of the most confounding characteristics, commonly seen in malignant, but even in benign melanocytic nevi, is represented by the regression phenomenon. The identification of regression, through dermoscopical observation, can be predictive of a tricky histopathological examination. Therefore, this feature should be an alert to a meticulous clinical, dermoscopical and histopathological correlation for correct analysis of melanocytic skin lesions. A 26-year-old man was referred to our department for a pigmented skin lesion localized on his trunk. It was clinically and dermoscopically diagnosed as atypical melanocytic nevus with central regression. After 1 year the lesion underwent considerable changes, leading to a nearly complete regression. The lesion was excised and, on the basis of clinical, dermoscopical and histopathological correlation, was interpreted as a junctional melanocytic nevus with regression. In our case the association of clinical, dermoscopical and histopathological experience, resulted an important and useful method, in order to proper interpret and correctly diagnose an atypical melanocytic skin lesion.

In November 2009, a 26-year-old man was referred to our department for a pigmented skin lesion on his trunk. The lesion appeared as a brown macule, sized $5 \mathrm{~mm}$ in diameter, with an irregular shape and no associated pruritus or discomfort. Family history of dysplastic nevi or melanoma was negative. Dermoscopic examination showed: reticular pattern with central regression, constituted by blue-white areas, and diffuse dots/globules (Figure 1a). A diagnosis of atypical melanocytic nevus with partial regression was made and excision of the lesion was recommended, but the patient did not show up, even after several reminders. He presented for a follow up after 1 year, when the lesion was almost totally disappeared. It was dermoscopically re-analyzed revealing diffuse white area with only a small central residual light brown pigmentation network (Figure 1b). The lesion was finally excised and histopathologic examination performed, diagnosing it as a melanocytic blue nevus. Since there was no concordance between histopathology, dermoscopy and clinical history the specimen was re-analyzed histopathologically. The examination confirmed the presence of fibrosis, neovascularization and heavily-pigmented dendritic melanocytes in the dermis (Figure 1c), but it showed also areas with irregular junctional melanocytic activity with a focal trend toward the upward spreading (pagetoid). The dermal component was constituted of melanocytes with small round nuclei, with rare nucleoli, and showed a solid growth pattern, with deep nodular areas characterized by high cellularity, without significant atypia (Figure 1d). This component showed a striking immunoreactivity for S-100 protein and ki-67 (MIB-1), but it was negative for HMB45. Based on the review of clinical, dermoscopical and histopathological features, the lesion was now diagnosed as a junctional melanocytic nevus with regression. A strict follow-up every 6 months was recommended.

The regression phenomenon represents one
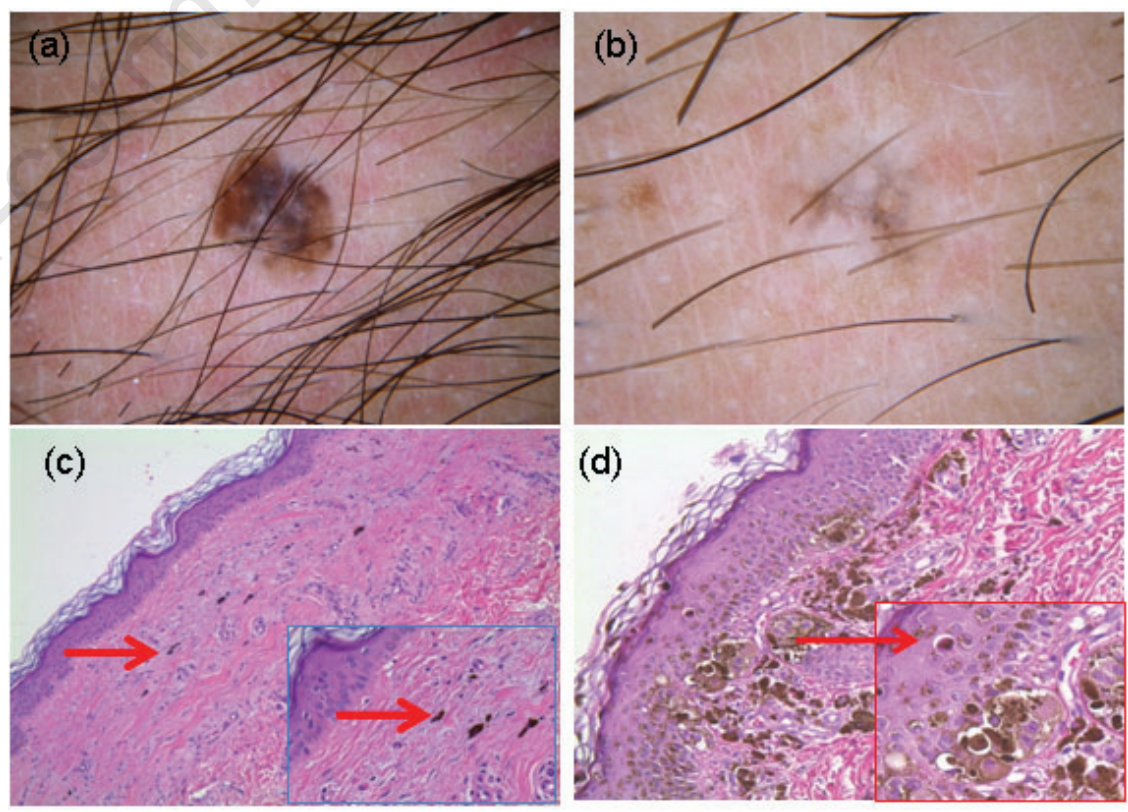

Figure 1. (a) Dermoscopic examination at the first observation and (b) after 1 year. Histopathological examination: (c) fibrosis, neovascularization and heavily-pigmented dendritic melanocytes in the dermis (arrows) (hematoxylin and eosin stain; H\&E, X1020 magnification); (d) irregular junctional melanocytic activity with a focal trend toward the upward spreading (pagetoid) (arrow). In the dermis presence of melanocytes with small round nuclei, rare nucleoli, and a solid growth pattern with high cellularity, but no significant atypia (hematoxylin and eosin stain; H\&E, X20-40). 
ones. Zalaudek et al. ${ }^{1}$ showed an absolute correspondence between the dermoscopic bluewhite structures and the presence of partial or focal regression histopathologically. Ackerman et $a .^{2}$ reported a dermoscopical - histopathological correlation between blue areas and the melanosis type of regression as well as between white areas and the fibrosis type of regression. Our case completely fitted with these findings.

In the last two decades several studies have been performed investigating the diagnostic impact of dermoscopy, demonstrating the increase of accuracy in diagnosing pigmented skin lesions compared with clinical examination by the naked eye. ${ }^{3,4}$ However, dermoscopy is not $100 \%$ accurate in differentiating melanocytic skin lesions as these entities frequently are characterized by 'overlapping' dermoscopic features. ${ }^{5,6}$ The loss of typical dermoscopic features over time, as in our case, represents a challenge for the dermatologist. Ferrara et al. ${ }^{7}$ showed that the presence of features as regression in melanocytic lesions might lead to an interobserver disagreement on diagnosis from a dermoscopical but also histopathological point of view. The knowledge of a dermoscopic pattern which might be predictive of a histopathological difficulty for the analysis of melanocytic skin lesions should be an alert to a meticulous clinical, dermoscopical and histopathological correlation. ${ }^{8}$ This report confirmed this correlation as an important and useful method in order to interpret and diagnose an atypical melanocytic skin lesion. In conclusion, we want to emphasize the important role of the interdisciplinary collaboration in the management of melanocytic skin moles.

\section{References}

1. Zalaudek I, Argenziano G, Ferrara G, et al. Clinically equivocal melanocytic skin lesions with features of regression: a dermoscopic-pathological study. $\mathrm{Br} \mathrm{J}$ Dermatol 2004;150:64-71.

2. Ackerman AB, Cerroni L, Kerl H. Pitfalls in Histopathologic Diagnosis of Malignant Melanoma. Philadelphia: Lea \& Febiger;1994.
3. Mayer J. Systematic review of the diagnostic accuracy of dermatoscopy in detecting malignant melanoma. Med J Aust 1997; 167:206-10.

4. Kittler H, Pehamberger H, Wolff K, Binder M. Diagnostic accuracy of dermoscopy. Lancet Oncol 2002;3:159-65.

5. Hofmann-Wellenhof R, Blum A, Wolf IH, et al. Dermoscopic classification of atypical melanocytic nevi (Clark nevi). Arch Dermatol 2001;137:1575-80.

6. Argenziano G, Scalvenzi M, Staibano S, et al. Dermatoscopic pitfalls in differentiating pigmented Spitz naevi from cutaneous melanomas. Br J Dermatol 1999;141:78893.

7. Ferrara G, Argenziano G, Soyer HP, et al. Dermoscopic and histopathologic diagnosis of equivocal melanocytic skin lesions: an interdisciplinary study on 107 cases. Cancer 2002;95:1094-100.

8. Ferrara G, Argenziano G, Soyer HP, et al. Histopathologic interobserver agreement on the diagnosis of melanocytic skin lesions with equivocal dermoscopic features: a pilot study. Tumori 2000;86:445-9. 